\title{
Excursion of the Rotator Cuff Under the Acromion
}

\section{Patterns of Subacromial Contact*}

\author{
Evan L. Flatow, †‡ MD, Louis J. Soslowsky, $\mathrm{PhD}$, Jonathan B. Ticker, $†$ MD, \\ Robert J. Pawluk, $†$ MS, Matthew Hepler, $\uparrow$ MD, Jon Ark, $†$ MD, Van C. Mow, $†$ PhD, and \\ Louis U. Bigliani, $†$ MD
}

\begin{abstract}
From the fOrthopaedic Research Laboratory, New York Orthopaedic Hospital, Columbia-Presbyterian Medical Center, New York, New York, and the §Orthopaedic Research Laboratories, the University of Michigan, Ann Arbor, Michigan
\end{abstract}

\begin{abstract}
Nine fresh-frozen, human cadaveric shoulders were elevated in the scapular plane in two different humeral rotations by applying forces along action lines of rotator cuff and deltoid muscles. Stereophotogrammetry determined possible regions of subacromial contact using a proximity criterion; radiographs measured acromiohumeral interval and position of greater tuberosity. Contact starts at the anterolateral edge of the acromion at $0^{\circ}$ of elevation; it shifts medially with arm elevation. On the humeral surface, contact shifts from proximal to distal on the supraspinatus tendon with arm elevation. When external rotation is decreased, distal and posterior shift in contact is noted. Acromial undersurface and rotator cuff tendons are in closest proximity between $60^{\circ}$ and $120^{\circ}$ of elevation; contact was consistently more pronounced for Type III acromions. Mean acromiohumeral interval was $11.1 \mathrm{~mm}$ at $0^{\circ}$ of elevation and decreased to $5.7 \mathrm{~mm}$ at $90^{\circ}$, when greater tuberosity was closest to the acromion. Radiographs show boneto-bone relationship; stereophotogrammetry assesses contact on soft tissues of the subacromial space. Contact centers on the supraspinatus insertion, suggesting altered excursion of the greater tuberosity may initially damage this rotator cuff region. Conditions limiting external rotation or elevation may also increase rotator cuff compression. Marked increase in contact with Type
\end{abstract}

\footnotetext{
*Presented at the annual meeting of the AOSSM, San Diego, California, July 1992.

$\ddagger$ Address correspondence and reprint requests to: Evan L. Flatow, MD, Florence and Herbert Irving Center for Clinical Research, $161 \mathrm{Ft}$. Washington Avenue, Room 247, New York, NY 10032.

No author or related institution has received any financial benefit from research in this study. See "Acknowledgments" for funding information.
}

III acromions supports the role of anterior acromioplasty when clinically indicated, usually in older patients with primary impingement.

The bursa and insertion of the supraspinatus on the greater tuberosity must repeatedly pass underneath the acromion with the arm in varying degrees of elevation and rotation during forceful overhead activity. Impingement of the rotator cuff and bursa by the coracoacromial arch is a common disorder in the shoulder. ${ }^{22,23,25,30}$ Subacromial bursitis as well as rotator cuff tendinitis and tears can result, leading to pain, weakness, and diminished athletic performance. Meyer ${ }^{19}$ implicated mechanical attrition under the acromion in the pathogenesis of rotator cuff lesions. Neer ${ }^{23}$ hypothesized that variation in acromial slope and morphology were clinically relevant, and Bigliani and coworkers ${ }^{4}$ demonstrated a relationship between acromial morphology and the incidence of rotator cuff tears in cadavers. The treatment of older patients with this type of impingement lesion by anterior acromioplasty and rotator cuff repair when needed has been highly effective. ${ }^{11}$ In the younger athletic shoulder, diagnosis of impingement and of associated injuries is more complex, and treatment regimens including surgery have been less effective.$^{17}$ Several factors have been associated with impingement lesions in the shoulder, including abnormal acromial morphol$0 \mathrm{gy},{ }^{4,5,23}$ intrinsic rotator cuff failure or tendinitis, ${ }^{26,40}$ and altered glenohumeral kinematics (e.g., instability). ${ }^{17} \mathrm{Un}$ fortunately, little or no quantitative data exist concerning normal patterns of contact between the acromion and the underlying cuff tissues and greater tuberosity, or abnormal contact patterns created by impingement. Such information is essential for proper diagnosis and treatment of impingement, especially in the athlete. 
Clinical tests for subacromial impingement frequently involve eliciting pain with elevation of the arm, accentuated with internal rotation; however, information concerning when, where, and how much contact occurs with such maneuvers is unavailable. Furthermore, anterior acromioplasty is a recognized treatment for symptomatic impingement. ${ }^{14,22,30}$ Better knowledge of which regions of the acromion are responsible for contact on the underlying rotator cuff might improve the rationale for and help optimize the technique of this procedure.

The acromiohumeral interval assesses acromion to humerus distance, rather than acromion to the rotator cuff tendon, and is measured on radiographs, which are twodimensional images of the three-dimensional acromiohumeral relationship. ${ }^{9,13,28,44}$ Furthermore, radiographic and other imaging techniques are generally performed at $0^{\circ}$ of elevation rather than positions where impingement is clinically observed. In the current study, an optical stereophotogrammetry technique ${ }^{2,37}$ is used to determine the contact areas at the subacromial articulation. ${ }^{39}$ Range of motion is produced in vitro by simulation of the coordinated actions of the four rotator cuff muscles and three heads (anterior, middle, and posterior) of the deltoid muscle, without disrupting the integrity of the bursa or glenohumeral joint capsule. The clinical value, as well as the limitations, of radiographs were assessed by comparing the three-dimensional maps obtained using the stereophotogrammetric technique with measurements of acromiohumeral distances on high-quality, standardized radiographs of the cadaveric shoulder in corresponding positions.

\section{MATERIALS AND METHODS}

\section{Specimens and specimen preparation}

Nine fresh human cadaveric shoulders (average age, 73 years) were used in this study. Standard AP and lateral radiographs in the plane of the scapula were obtained of each specimen in $0^{\circ}$ of elevation, and only shoulders without evidence of arthritis, fractures, or other abnormalities were used in this study. Specimens were stored at $-20^{\circ} \mathrm{C}$ until the day before testing. Each specimen was thawed overnight at room temperature in preparation for dissection. Subsequently, each specimen was dissected free of all soft tissue, leaving intact only the deltoid, rotator cuff tendons, glenohumeral joint capsule, and the coracoacromial and coracohumeral ligaments. Specimens with rotator cuff tears were excluded. During storage, dissection, preparation, and testing, each specimen was preserved in gauze moistened with physiologic Ringer's solution containing protease inhibitors to retard specimen dehydration and degradation.

The tendinous insertion of each rotator cuff muscle was exposed by dissection. Because the infraspinatus and teres minor muscles have similar functions, and because they share adjacent insertion sites, a single line of action for their resultant force was used. Loads were applied onto the tendinous insertions of the supraspinatus, subscapularis, and infraspinatus and teres minor muscles via a sutured loop made of high tensile strength woven Dacron. Flexible cables were fastened to the Dacron loops, and each cable was positioned through the center of each muscle's physiologic cross-sectional area.

Dissection of the deltoid muscle allowed isolation of its anterior, middle, and posterior heads, including their associated origins and insertions. The deltoid muscle was subsequently resected, and bolts were placed through the humeral shaft cortex at each of the three insertion sites (Fig. 1). An eye screw was fixed to the coracoid at the origin of the anterior deltoid at the clavicle. Eye screws were also placed in the lateral acromial edge and the scapular spine to establish the line of action of the middle and posterior deltoid muscles, respectively. Flexible cables were attached to the humeral cortical bolts and threaded through their corresponding eye screws to simulate the force acting on each of the three deltoid heads (Fig. 1). Finally, each of the six cables, simulating the deltoid heads and rotator cuff muscles, were attached to a turnbuckle in series with a calibrated spring scale.

\section{Specimen positioning}

A Kirschner wire was used as a reference for humeral shaft rotation. The wire was placed distal to the deltoid tuberosity and parallel to the condylar axis before the resection of the humeral condyle. The lower third of the scapula was then embedded with $20^{\circ}$ of anterior tilt and the medial border vertically aligned to approximate the anatomic orientation. Codman ${ }^{7}$ noted that maximum elevation of the arm can only be achieved at a fixed degree of external rotation. Therefore, to obtain subacromial contact over the largest possible range of elevation, each humerus was manually elevated to determine the unique (individual) degree of external rotation for each shoulder that would allow for maximum elevation of the arm. This angle of rotation, measured between the scapular plane and the epicondylar axis, was defined as the "starting rotation" and averaged $40^{\circ} \pm 8^{\circ}$ of external rotation for the specimens in the current study.

The ranges of applied muscle loads were predetermined and selected based on clinical, anatomic (physiologic

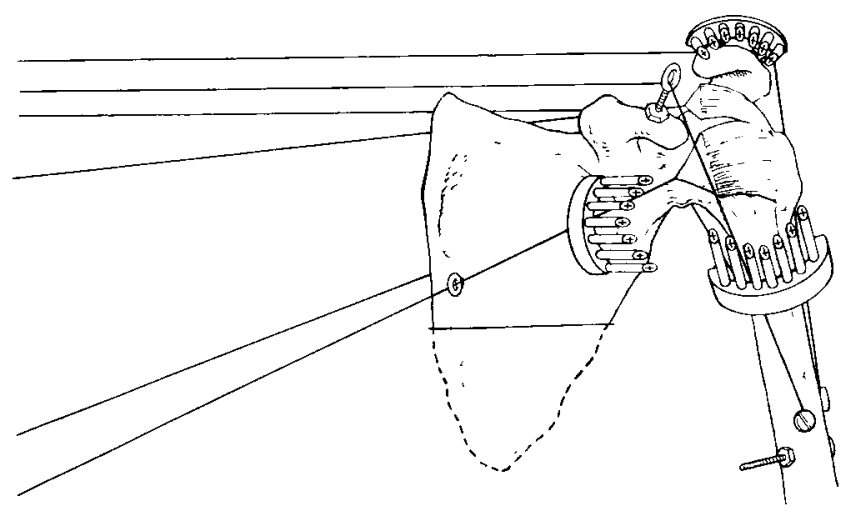

Figure 1. Humeral elevation and rotation were achieved by simulated muscle forces. The rotator cuff tendons were sewn to woven Dacron strips, to which tensile loads were applied, and the three heads (anterior, middle, and posterior) of the deltoid muscle were simulated through cables along their line of actions. 
cross-sectional area), mechanical (lever arms), and electromyographic data. ${ }^{27,36}$ Thus, loading each muscle insured position control of the humerus, yielding a physiologically reasonable solution for the statically indeterminate problem. ${ }^{36,38}$ The starting rotation, as defined above, was maintained while the arm was elevated in the scapular plane by coordinating the cable tensions of each muscle. For the stereophotogrammetric studies, the arm was elevated in $30^{\circ}$ increments from $0^{\circ}$ until maximum elevation was attained. The arm was also elevated in the scapular plane at a humeral rotation $20^{\circ}$ internally rotated from the starting rotation. To simulate arm weight, a $3.2-\mathrm{kg}$ mass was attached at a fixed distance from the center of rotation of the humeral head. The scapulothoracic motions contributing to total arm elevation were determined from previous studies. ${ }^{10,12,29}$ To compensate for the fixed scapula, the line of action of the force applied to the humerus to simulate arm weight (and the direction of gravity) was adjusted to reflect physiologic scapular tilt through the range of arm elevation. ${ }^{10,12,29}$ This procedure results in an equivalent situation to rotating the scapula but is experimentally much simpler.

\section{Stereophotogrammetry}

Stereophotogrammetry is defined as the science of obtaining precise dimensional measurements of an object using mathematical computations through the process of recording, measuring, and interpreting pairs of photographic images. A mathematical model is then constructed to represent relations between points in three-dimensional space and their corresponding two-dimensional images on the photographs. This procedure might be thought of as analogous to the process by which the human brain processes two images, one from each eye, to obtain depth perception. Stereophotogrammetry is used for many applications. One common method of mapping the surface contour of the earth uses photographs of an area taken with a precision camera mounted on an airplane flying over the region of interest. In the current study, this procedure is applied to the determination of contact areas in the subacromial space, as will be described below.

The stereophotogrammetric method was used to determine the subacromial contact areas. ${ }^{38,39}$ To do this, a semicircular set of seven precision optical alignment targets was rigidly fixed to the proximal humerus, and another set fixed to the acromion using threaded Steinmann pins and polymethylmethacrylate. Each instrumented shoulder specimen was then placed within a custom-built calibration frame, and a stereogram (pair of convergent photographs) was taken of the two sets of targets using a largeformat camera at each shoulder position of the experiment (Fig. 2).

Each shoulder specimen was disarticulated after testing through the full range of motion as described above. Subsequently, each portion of the dissected joint was placed within the calibration cage, and a fine optical grid was projected onto the articular surface to provide recognizable surface points for digitization. Similar stereograms were made of the undisturbed rigidly fixed optical targets and
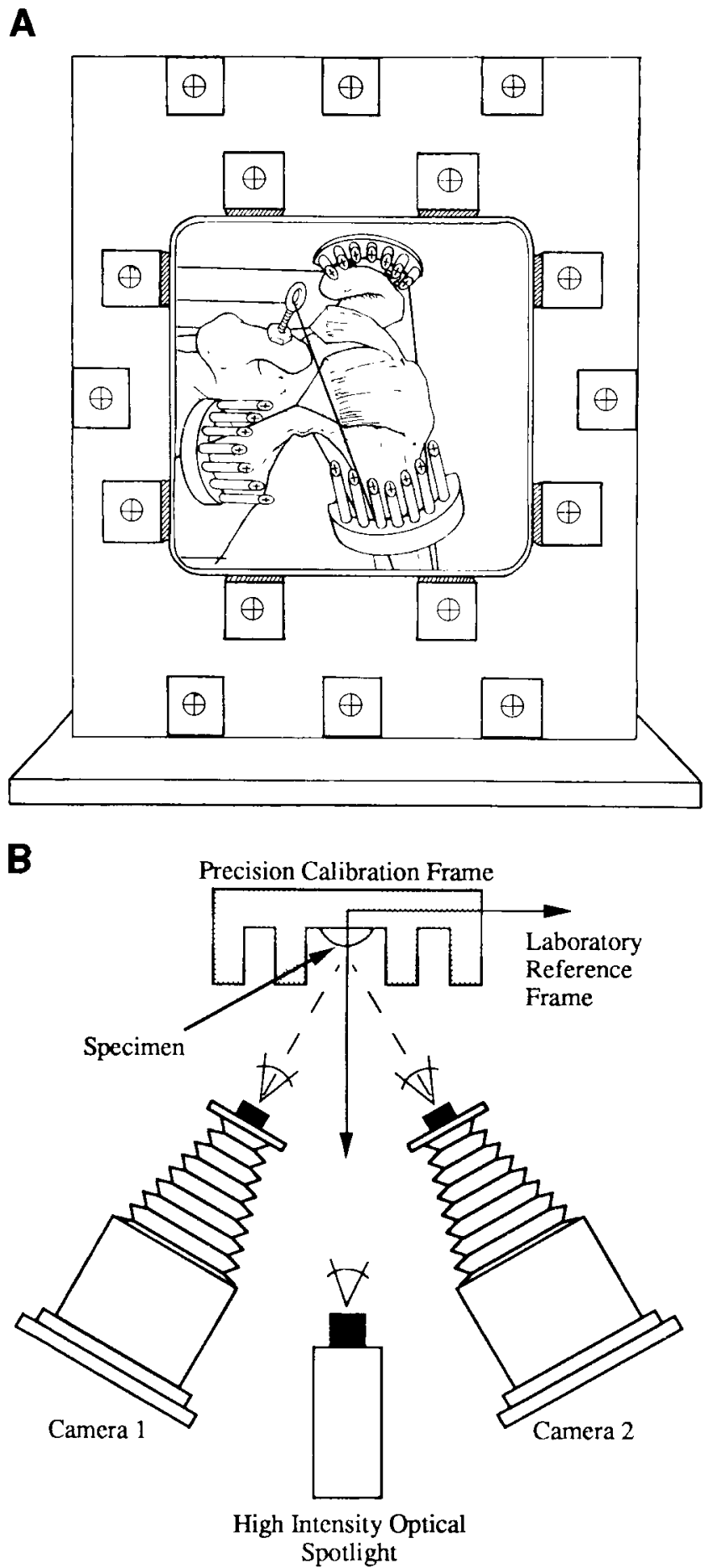

Figure 2. A, three rigid sets of optical alignment targets are affixed to the shoulder, one each to the proximal humerus, scapula (not used for this study), and acromion. The shoulder, positioned by simulated muscle forces, is placed in a calibration frame. B, stereograms of the shoulder, targets, and frame are taken.

the undersurface of the acromion. The surface and target points on each photograph were digitized using a high-accuracy two-dimensional digitizer, and the three- 
dimensional coordinates of each point were reconstructed. ${ }^{2,37}$ This procedure provides accurate quantitation of the geometry of the soft tissues overlying the proximal humerus and of the undersurface of the acromion, relative to their respective optical targets (Fig. 3). The digitized stereophotogrammetric surfaces are then curve-fitted with bicubic patches to create a continuous three-dimensional computer graphics representation of each joint surface (Fig. 4). Using the three-dimensional coordinates of the precision optical targets, exact amounts of translation and rotation may be calculated to reposition the acromial and supraspinatus tendon surfaces to their original positions and orientations within the intact joint. ${ }^{36,37,39}$ At regions of overlap, the rotator cuff tendon surface would be expected to deform..$^{33,36,38}$ Areas of proximity between the subacromial surface and the superior surface of the supraspinatus tendon provide an indication of the regions where contact may occur. Although the tendon may not be compressed at such regions in these normal cadaveric specimens, these regions are the most likely to be in contact in the presence of anatomic or kinematic abnormalities.

\section{Radiographic analysis}

For the radiographic portion of this study, seven of the shoulder specimens from the stereophotogrammetric study (before disarticulation) and one additional fresh-frozen cadaveric shoulder were taken though a range of motion with the same muscle simulation technique described above. High-quality AP radiographs were taken with the beam oriented perpendicular to the plane of the scapula at a fixed distance in a standardized fashion. In addition, outlet views (lateral views of the scapula with the beam aimed down the scapular spine) were taken in each position for a qualitative comparison with the AP views, and to profile acromial morphology. ${ }^{4,5,24}$ Rotation was maintained at the starting rotation, and radiographs were taken at $0^{\circ}$ of arm elevation, and in $10^{\circ}$ increments from $60^{\circ}$ to $120^{\circ}$ of arm elevation in the scapular plane.

The acromiohumeral interval for each AP radiograph in each incremental position was determined using a

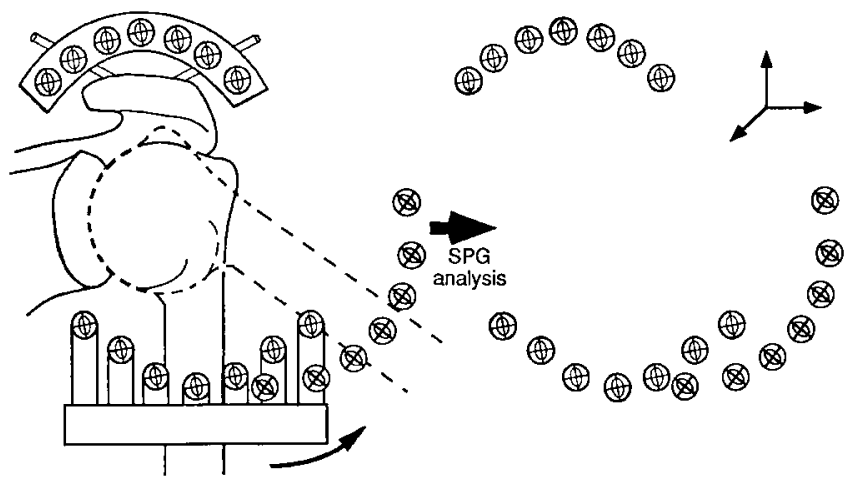

Figure 3. Stereograms are taken as the humerus is elevated in ir.crements. By stereophotogrammetric (SPG) analysis (arrow), the three-dimensional relationship between the optical markers affixed to each bone is determined at each position of elevation.

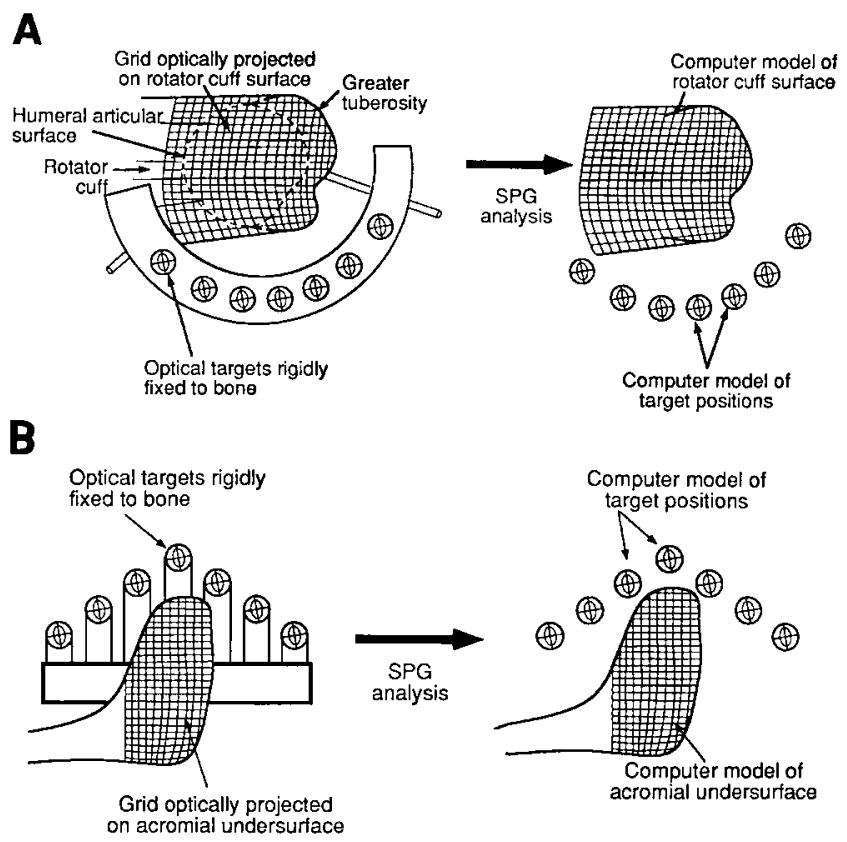

Figure 4. A, after the whole-joint model is tested in multiple positions, the joint is disarticulated, and the three-dimensional geometry of the surface of the rotator cuff and proximal humerus, and the relationship of these structures to the affixed targets, are determined using stereophotogrammetry (SPG) analysis (arrow). B, the three-dimensional geometry of the acromion and its relationship to the affixed target are similarly determined.

precision caliper. Distances were measured from the midpoint of the anterior acromion (located between its lateral edge and the acromioclavicular joint, at the level of the inferior cortex) to the point directly below on the proximal humerus (Fig. 5). A second measurement was taken from the midpoint of the anterior acromion to the crest of the greater tuberosity.

\section{RESULTS}

Relative position of the undersurface of the acromion and of the proximal humerus was determined using stereophotogrammetry for each shoulder at all positions tested. Results were highly dependent on the degree of arm elevation (Table 1). Specifically, at middle elevation, more shoulders demonstrated contact than at either of the extremes. In addition, when $20^{\circ}$ internally rotated from the starting rotation, more shoulders were in contact than when tested at the starting rotation.

Regions of proximity, defined as all points on one surface that were within $5 \mathrm{~mm}$ of the other surface, were also investigated. These results demonstrated consistent trends and support the concept that the humerus and undersurface of the acromion are closest to each other in the range of $60^{\circ}$ to $120^{\circ}$ of arm elevation (Table 2).

Contact and proximity were located at the anterolateral edge of the acromion at $0^{\circ}$ elevation and shifted medially to the anteromedial edge with increasing arm elevation 


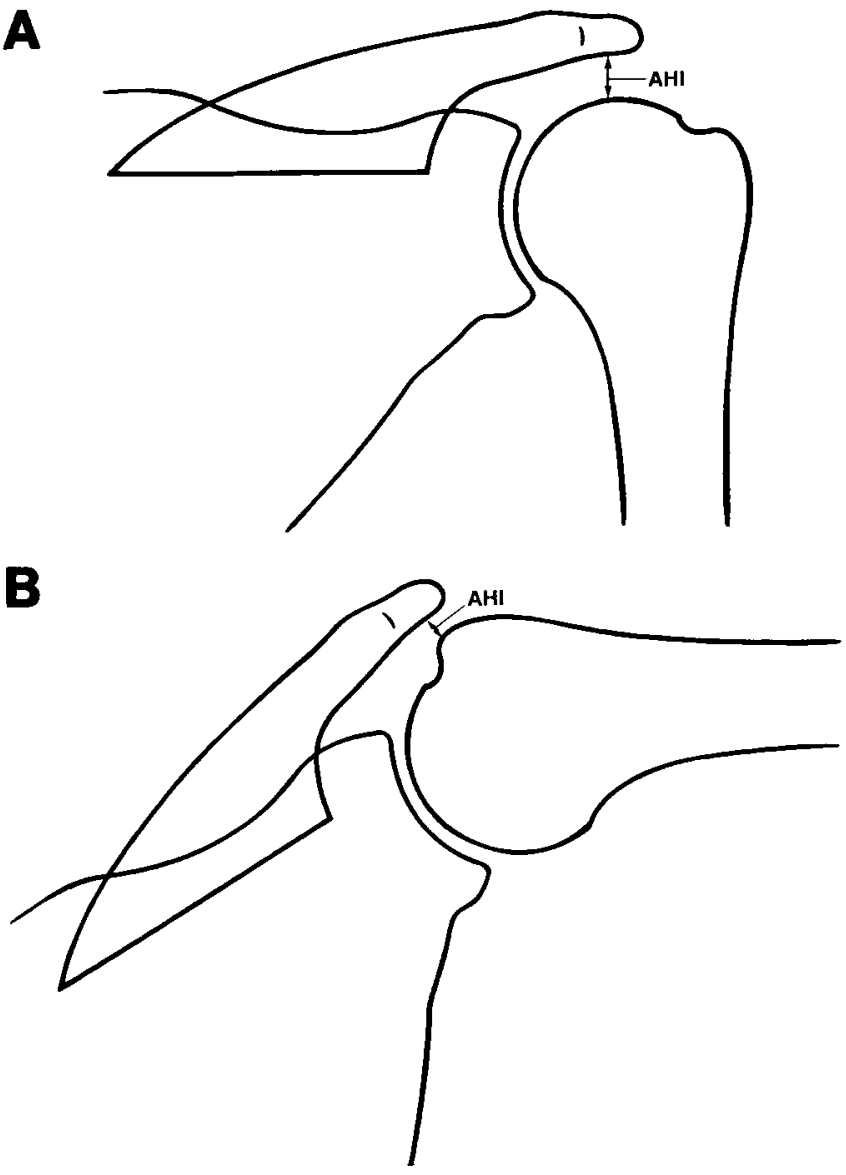

Figure 5. A, diagram of measurements taken of the acromiohumeral interval $(\mathrm{AHI})$ on $\mathrm{AP}$ radiographs. Here, the interval includes the thickness of the rotator cuff tendon. $B$, in the measurement of $\mathrm{AHI}$ in elevation, the bone of the greater tuberosity is directly below the acromion, and the interval no longer accommodates the rotator cuff tendon.

TABLE 1

Number of shoulders demonstrating subacromial contact (overlap) at various positions

\begin{tabular}{|c|c|c|}
\hline $\begin{array}{c}\text { Elevation } \\
\text { angle } \\
\text { (deg) }\end{array}$ & $\begin{array}{l}\text { No. of shoulders } \\
\text { in contact at } \\
\text { starting rotation }\end{array}$ & $\begin{array}{c}\text { No. of shoulders } \\
\text { in contact at } 20^{\circ} \\
\text { internal to starting rotation }\end{array}$ \\
\hline 0 & 2 & 5 \\
\hline 30 & 5 & 7 \\
\hline 60 & 7 & 9 \\
\hline 90 & 6 & 9 \\
\hline 120 & 2 & 4 \\
\hline 150 & 1 & 0 \\
\hline 180 & 0 & 0 \\
\hline
\end{tabular}

(Fig. 6). In each case, and at all positions tested, contact involved only the anterior portion of the acromion. On the humeral side, contact and proximity were located at the proximal end of the biceps region and supraspinatus tendon at $0^{\circ}$ of elevation and shifted distally along these tendons with increasing arm elevation. In the range of $60^{\circ}$ to $120^{\circ}$ of arm elevation, contact was focused at the supraspinatus insertion. When external rotation is decreased, a subtle distal and posterior shift was noted in proximity on
TABLE 2

Acromiohumeral proximity areas ${ }^{\circ}$

\begin{tabular}{ccc}
\hline $\begin{array}{c}\text { Elevation angle } \\
(\mathrm{deg})\end{array}$ & $\begin{array}{c}\text { Areas at starting } \\
\text { rotation }\left(\mathrm{cm}^{2}\right)\end{array}$ & $\begin{array}{c}\text { Areas at } 20^{\circ} \text { internal } \\
\text { to starting rotation } \\
\left(\mathrm{cm}^{2}\right)\end{array}$ \\
\hline $\mathbf{0}$ & $1.1 \pm 1.1$ & $1.4 \pm 1.2$ \\
$\mathbf{3 0}$ & $2.3 \pm 1.9$ & $2.2 \pm 1.5$ \\
$\mathbf{6 0}$ & $4.3 \pm 2.3$ & $3.2 \pm 2.0$ \\
$\mathbf{9 0}$ & $4.6 \pm 1.4$ & $3.0 \pm \mathbf{1 . 4}$ \\
$\mathbf{1 2 0}$ & $3.3 \pm 1.9$ & $2.0 \pm \mathbf{1 . 7}$ \\
$\mathbf{1 5 0}$ & $2.7 \pm 1.7$ & $1.0 \pm 0.9$ \\
$\mathbf{1 8 0}$ & $1.3 \pm \mathrm{NA}^{b}$ & $0.8 \pm 0.2$ \\
\hline
\end{tabular}

a Data reported as means \pm standard deviations.

${ }^{b}$ Not applicable.

the tendons covering the humeral head (Fig. 7). Furthermore, contact and proximity were consistently more pronounced for shoulders with Type III acromions compared with those with Type I or II acromions (Fig. 8).

For radiographic evaluation, the apparent inferior acromial cortex was used to measure the acromiohumeral interval on the AP radiograph, as established from prior studies., ${ }^{9,44}$ It was seen as a radio-dense line corresponding to the portion tangent to the x-ray beam of the curved inferior acromial cortex. This line was, therefore, not a constant anatomic landmark but an artifact dependent on the orientation of the $\mathrm{x}$-ray beam and the morphology of the acromion. The point "directly below" on the humerus as seen on the radiograph is not necessarily directly below in three-dimensional space but rather might be somewhat anterior or posterior. The outlet radiograph exhibited the anteroposterior curve of the acromion and humerus well but superimposed them mediolaterally. This made it difficult to tell whether the greater tuberosity or the more medial humeral articular surface was directly under the acromion. The AP and outlet radiographs for the same shoulder in the same position allowed some judgment of these relationships, but in the absence of discrete markers for comparison, only qualitative estimates were possible. Nevertheless, our technique is similar to those reported in other studies done only at $0^{\circ}$ of elevation,,$^{9,13,44}$ and it thus allows the findings from those studies to be compared with our results at different elevations in the AP plane. Also, because visual inspection of the subacromial interval on radiographs is commonly employed clinically, it was believed to be important to examine this parameter in our cadaveric model.

The acromiohumeral interval for all shoulders at $0^{\circ}$ of elevation was between 9 and $14 \mathrm{~mm}$ (average, 11.1) (Table 3 ). With arm elevation from $0^{\circ}$ to $110^{\circ}$, the acromiohumeral interval decreased continuously and measured an average of $4.7 \mathrm{~mm}$ at $110^{\circ}$, a decrease of $57 \%$. Below $90^{\circ}$ of elevation, the articular surface of the humerus was directly below the acromion. Thus, the acromiohumeral interval represented the bone-to-bone interval accommodating the soft tissues, including the humeral articular cartilage capsule, rotator cuff tendon, bursa, and acromial periosteum. At $90^{\circ}$ of elevation, the crest of the greater tuberosity passed below the acromion at its closest proximity (average, $5.8 \mathrm{~mm}$ ) (Fig. 9). With further elevation, the lateral portion of the greater tuberosity was in closest proximity to the acromial 


\section{A $\quad 0^{\circ}$ elevation in S.R.}

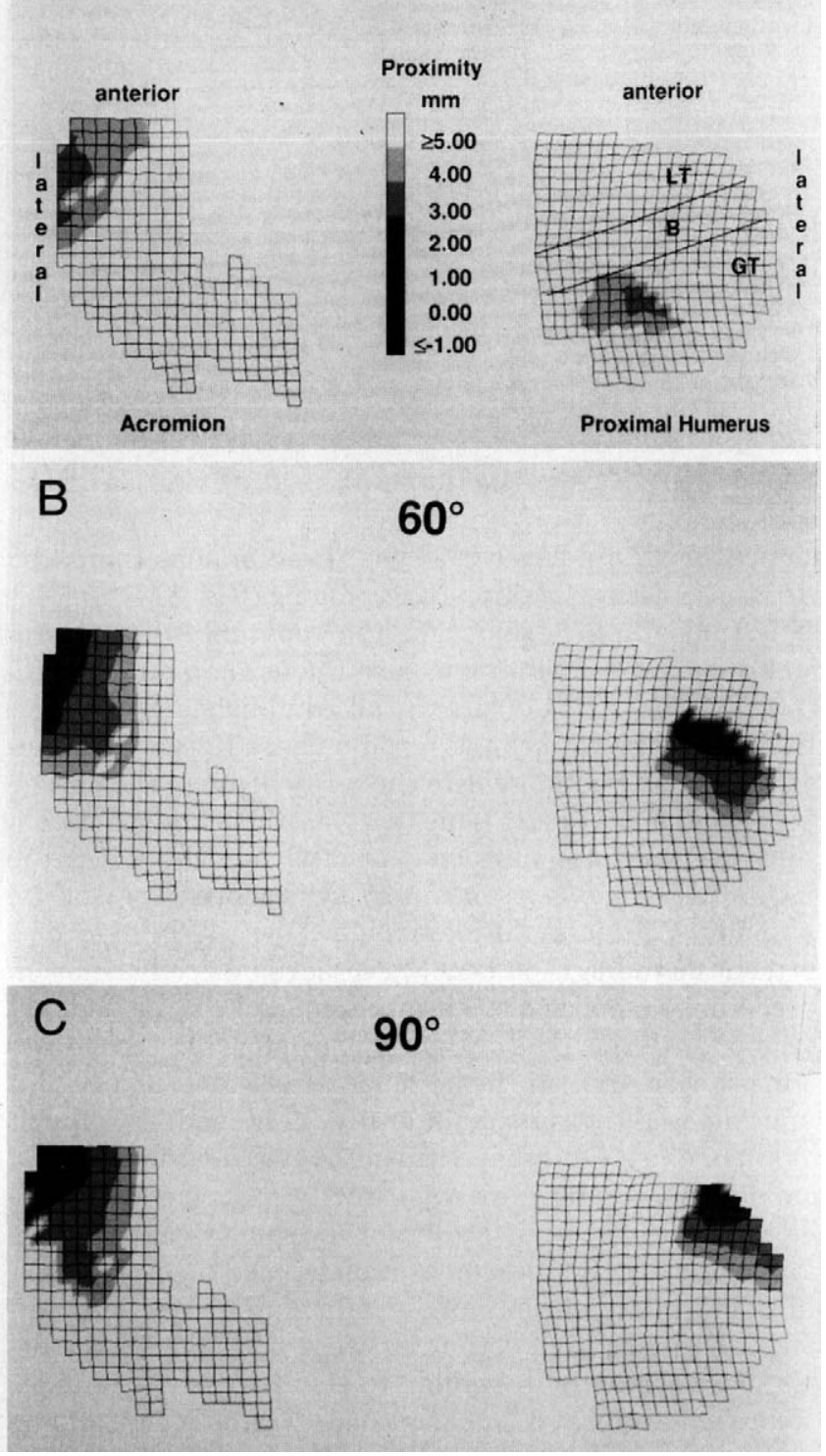

Figure 6. A, subacromial contact patterns for a right shoulder at $0^{\circ}$ of arm elevation in the starting rotation (SR). Gray levels represent proximity of one surface to the other in millimeters: (B) biceps tendon region, (GT) greater tuberosity, (LT) lesser tuberosity. B, the same shoulder at $60^{\circ}$ of elevation. $\mathrm{C}$, the same shoulder at $90^{\circ}$ of elevation.

undersurface because the cuff insertion and tuberosity had already passed medially. Therefore, beyond $90^{\circ}$ of elevation, the acromiohumeral interval no longer accommodated the articular cartilage or cuff tendon but only the periosteum of the acromion and greater tuberosity.

The radiographic investigation did not evaluate a position $20^{\circ}$ less externally rotated than the starting rotation in all shoulders. However, selected outlet views demonstrated that relative internal rotation tended to bring the greater tuberosity forward, directly under the anterior acromion, while relative external rotation allowed the

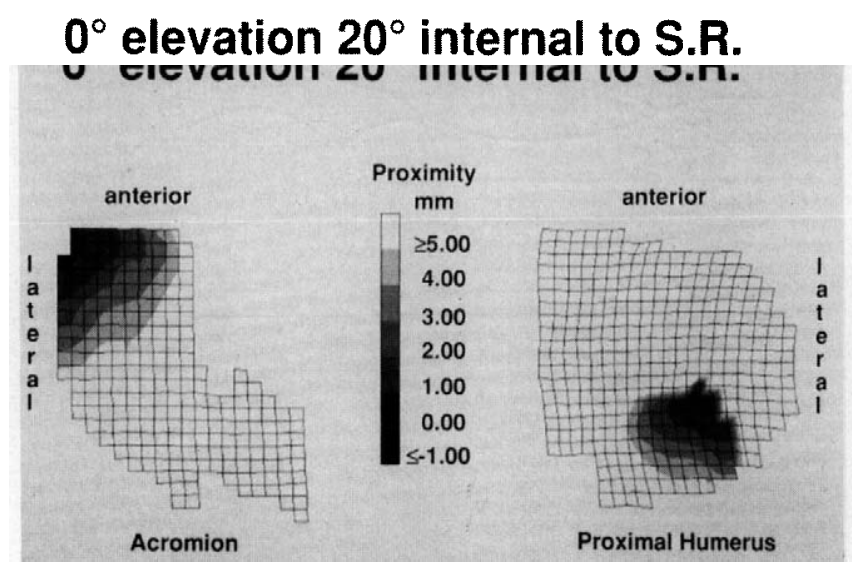

Figure 7. With a $20^{\circ}$ internal rotation from the starting rotation (SR), contact on the tendons covering the humeral head is shifted distally and posteriorly (compare with Figure 6A).

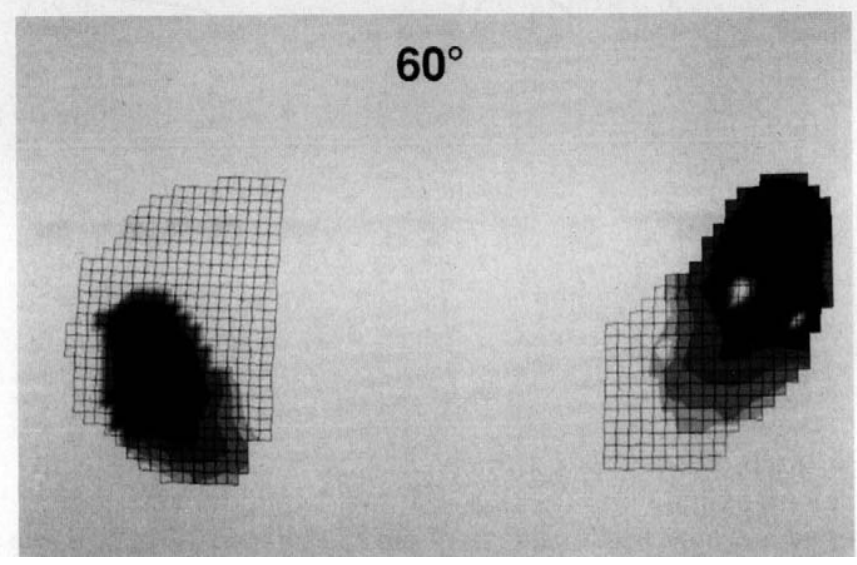

Figure 8. Subacromial contact pattern for a left shoulder with a Type III acromion at $60^{\circ}$ of arm elevation in the starting rotation. Note the increased contact on these surfaces as compared with Figure 6B.

TABLE 3

Measurements of anteroposterior radiographs ${ }^{a}$

\begin{tabular}{crc}
\hline Arm position (deg) & AHI (mm) & $\begin{array}{c}\text { Acromion to } \\
\text { greater tuberosity }(\mathrm{mm})\end{array}$ \\
\hline 0 & $11.1 \pm 1.4$ & $33.1 \pm 4.9$ \\
60 & $8.1 \pm 2.1$ & $11.8 \pm 4.7$ \\
70 & $7.5 \pm 2.0$ & $9.1 \pm 3.4$ \\
80 & $6.8 \pm 2.3$ & $6.6 \pm 1.9$ \\
90 & $5.7 \pm 2.4$ & $5.8 \pm 2.4$ \\
100 & $4.9 \pm 2.2$ & $7.1 \pm 3.1$ \\
110 & $4.7 \pm 2.1$ & $9.4 \pm 3.6$ \\
120 & $4.8 \pm 2.5$ & $11.7 \pm 3.7$ \\
\hline
\end{tabular}

${ }^{a}$ Radiographic measurements for the acromiohumeral interval (AHI) and distance from the midpoint of the acromion to greater tuberosity for all arm positions studies $(N=8)$.

tuberosity to pass more posteriorly (Fig. 10). This suggests that in some cases the two-dimensional AP radiographs may appear to demonstrate the tuberosity directly beneath the anterior acromion, when in fact it is more posterior and not in proximity. 

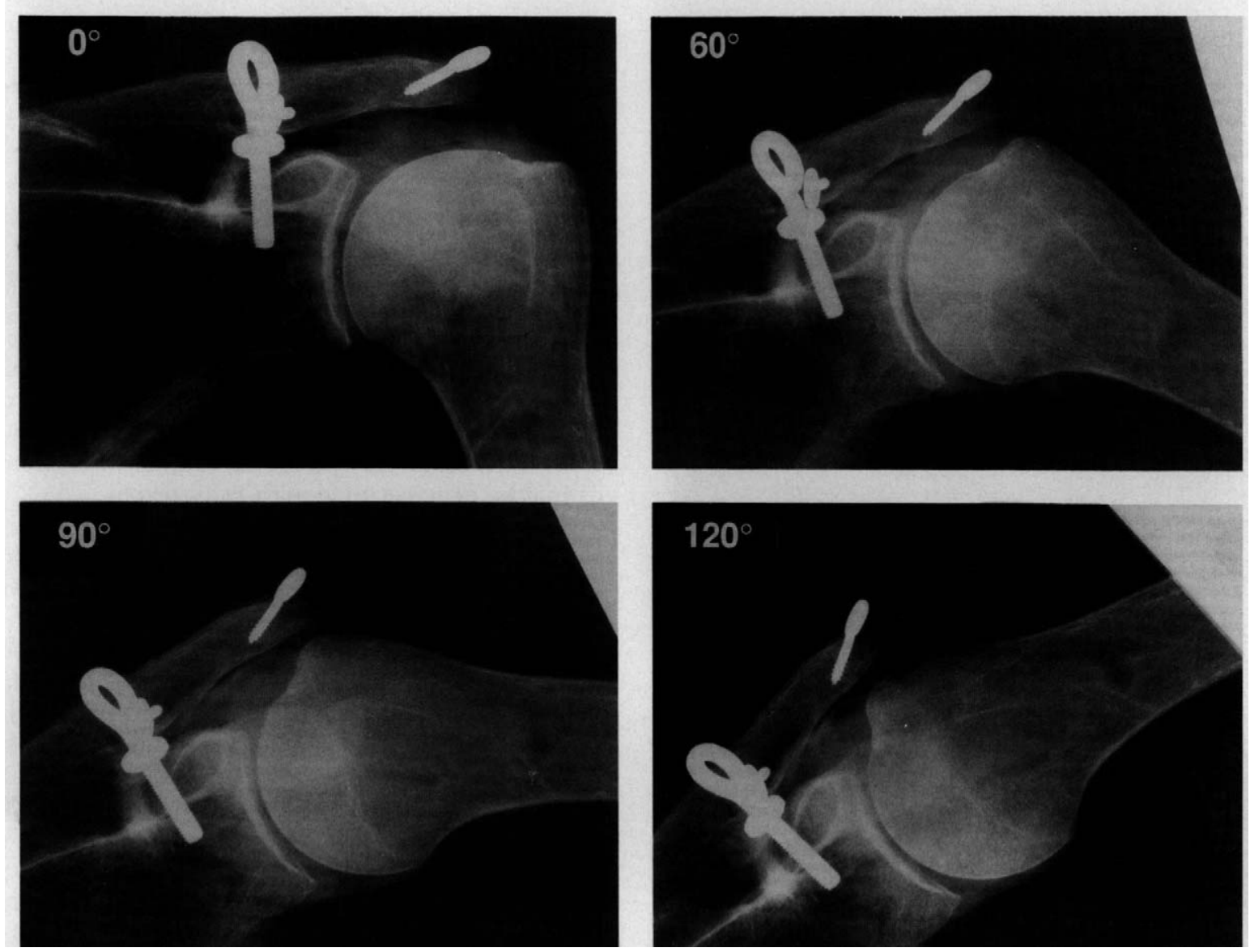

Figure 9. Anteroposterior radiographs in $0^{\circ}, 60^{\circ}, 90^{\circ}$, and $120^{\circ}$ of elevation in the starting rotation.

\section{DISCUSSION}

Meyer ${ }^{19}$ implicated mechanical attrition under the acromion in the pathogenesis of rotator cuff degeneration. Watson-Jones ${ }^{43}$ noted that pain may be produced when the acromion impinges on the rotator cuff tendon and recommended acromionectomy when surgical treatment was indicated. Others proposed lateral acromionectomy to remove the region of the acromion that seemed, on an AP radiograph, most likely to impinge on the elevating humerus. ${ }^{1,35}$ This two-dimensional view was challenged in 1972 by Neer, ${ }^{23}$ who pointed out that the humerus was not centered under the acromion, as suggested by AP radiographs, but was more anterior, under the anterior acromion, coracoacromial ligament, and acromioclavicular joint. In his report, Neer demonstrated a characteristic ridge of spurs and excrescences on the undersurface of the anterior aspect of the acromion. He thought that it was this anatomic region, and not the lateral or posterior acromion, that was responsible for impingement and introduced anterior acromioplasty: resection of the undersurface of the anterior acromion to decompress the underlying rotator cuff tendons. The current study confirms that regions of contact are focused on the anteroinferior acromion, supporting removal of this region in the surgical treatment of the impingement syndrome. Anterior acromioplasty is now a well-established treatment for rotator cuff impingement syndrome $\mathrm{e}^{14,22,23,30}$ and has recently been shown to reduce subacromial pressure as measured with a balloon manometer. ${ }^{31}$ However, in the young athletic shoulder subjected to repetitive, stressful overhead use, factors contributing to rotator cuff lesions and bursitis are complex, and the diagnosis and treatment of impingement lesions remain a challenge. ${ }^{17,42}$ Despite these problems, there is little information available on regions of subacromial contact on the underlying soft tissues. Such information could aid in the development and refinement of surgical treatment strategies.

Previous studies have provided limited information on the articulating mechanics of the subacromial region. With the $\operatorname{arm}$ in $0^{\circ}$ of elevation and neutral rotation, it has been shown that the distance between these two surfaces (acromiohumeral interval) as measured on AP radiographs is between 6 and $14 \mathrm{~mm}$ in normal subjects ${ }^{9,13}$ and is $6 \mathrm{~mm}$ or less in $50 \%$ of patients with rotator cuff tears. ${ }^{44}$ However, it must be emphasized again that these reported values represent the length of lines drawn on two-dimensional radiographs, onto which have been projected the threedimensional subacromial relationships. The stereophotogrammetry used in this study accurately maps these distances in three-dimensions. Furthermore, measurements 

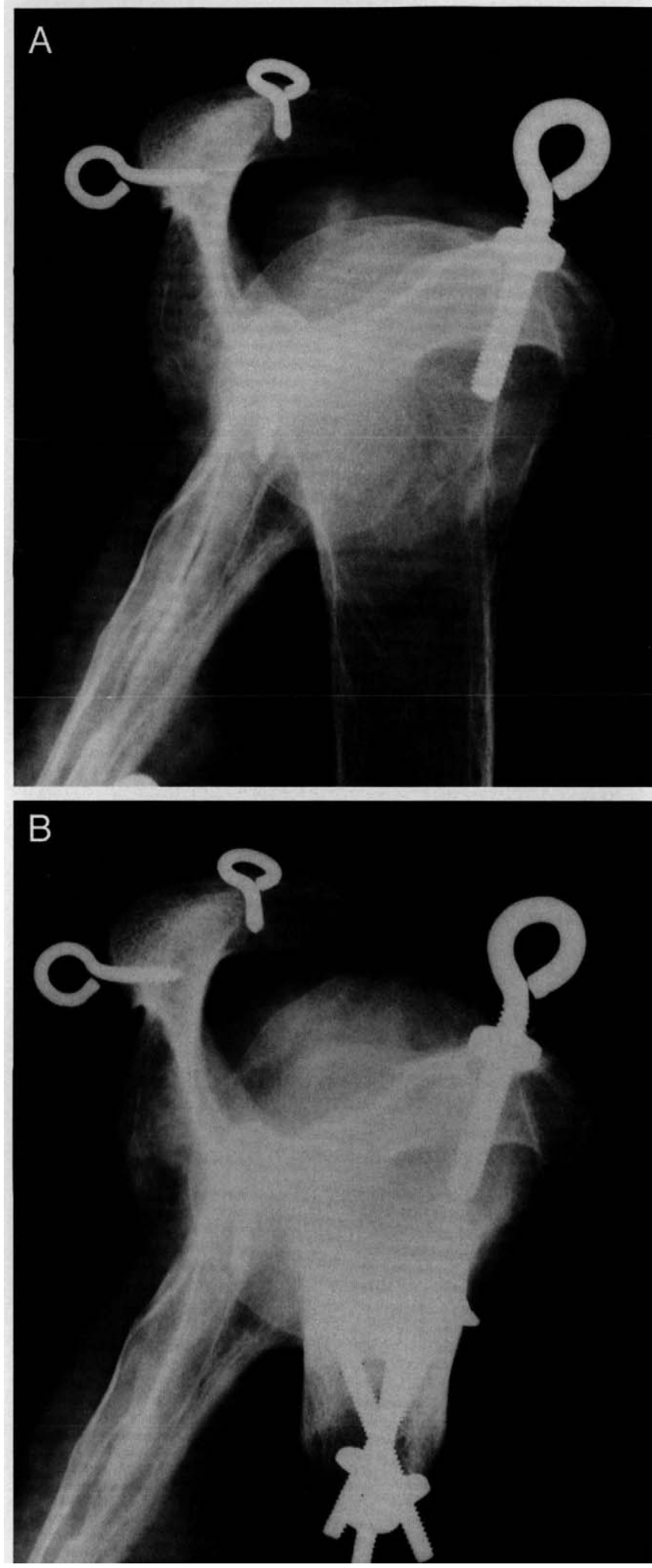

Figure 10. A, outlet radiograph in $0^{\circ}$ of elevation. These highdefinition radiographs of specimens stripped of all other tissues demonstrate the soft tissue shadow of the rotator cuff tendon. This is not seen well on clinical radiographs. Proximity of the anterior acromion to the tendon can be appreciated. B, outlet view in $90^{\circ}$ of elevation shows the greater tuberosity has rotated under the acromion. obtained from radiographs represent distances measured from bone to bone. They do not represent the distance from the articulating soft tissue that covers the humeral head (especially the rotator cuff tendons) to the soft tissue covering the undersurface of the acromion, as measured using stereophotogrammetry. Fluoroscopic examinations of a series of patients with torn rotator cuffs revealed bony contact between the greater tuberosity and a spur on the undersurface of the acromion that in some cases produced pain. ${ }^{8}$ However, in other patients with pain, no contact between bony surfaces was observed. An explanation for this is suggested by the results of the stereophotogrammetric portion of this investigation. The soft tissue covering the humeral head may, indeed, have been in contact with the undersurface of the acromion, causing symptoms that cannot be demonstrated with radiographs.

Most clinical studies report impingement occurring in the range of $60^{\circ}$ to $120^{\circ}$ of arm elevation. ${ }^{23,43}$ Despite the importance of understanding the relationship between the tendons and undersurface of the acromion through ranges of motion, limited information is available for positions other than at $0^{\circ}$ of elevation. Both stereophotogrammetric and radiographic data demonstrate that the region of closest proximity of the acromial undersurface and the cuff insertion or greater tuberosity or both is maximal in the middle range of elevation. Beyond this, the tuberosity clears the acromion and passes medially, in the normal shoulder. Therefore, provocative tests with the arm in full elevation are unlikely to represent maximum compression of the cuff under the acromion, which has greatest contact at the middle range of elevation. Recent work has suggested that as the arm fully elevates, the undersurface of the supraspinatus tendon can come into contact with the glenoid rim and that this may be a source of pain and tendon damage (Refs. 18, 41; C. M. Jobe, unpublished data, 1990). This mechanism was not investigated in the current study.

Burns and Whipple ${ }^{6}$ made qualitative visual observations of subacromial contact in five articulated cadaveric shoulders. They found that contact by the anterior tip of the acromion on the supraspinatus tendon and greater tuberosity was greatest in the middle ranges of humeral elevation. Nasca and coworkers ${ }^{21}$ applied a green dye to the inferior acromial surface and manually compressed the shoulder to produce contact at two discrete positions of $45^{\circ}$ and $90^{\circ}$ of abduction. They determined that contact areas increased from $45^{\circ}$ to $90^{\circ}$ of elevation. This result is consistent with the findings of Jerosch and coworkers ${ }^{16}$ in 1989, who used simulated muscle forces similar to those used in our current study. They found that subacromial pressure, measured with pressure-sensitive film, was highest at $90^{\circ}$ of abduction. However, this film was interposed between the acromion and rotator cuff, and, thus, it may have caused alterations in the contact areas and pressures being measured. By contrast, the optical stereophotogrammetric technique in this study allows measurements to be made without disrupting the joint capsule or subacromial space. This method accurately assesses gross migrations of contact patterns as well as minute shifting of areas. In addition, the stereophotogrammetric technique provides a 
precise and simultaneous determination of joint contact areas of both the glenohumeral and subacromial articulations of a given shoulder at multiple positions under various static loading conditions. ${ }^{36,38,39}$

Acromial shapes have been classified into three categories (Type I, flat; Type II, curved; Type III, hooked) and correlated with the incidence of rotator cuff tears. ${ }^{4,20}$ In these cadaveric and patient studies, $70 \%$ to $80 \%$ of cuff tears were associated with Type III acromions, $20 \%$ to $30 \%$ with Type II acromions, and $0 \%$ to $3 \%$ with Type I acromions. The acromions in the current study were also classified according to the three types above, and, although a limited number of specimens existed in each category (one Type I, four Type II, and four Type III), the current study supports this result. Shoulders with Type III acromions consistently had increased contact compared with shoulders with other acromial types. Previous studies of the coracoacromial arch have documented variations but have been unable to demonstrate their practical effect on contact on the rotator cuff and humerus. ${ }^{32,45}$ Anterior acromioplasty has generally been reserved for older patients with primary impingement, and the marked increase in contact with Type III acromions seen in these older cadavers would seem to support that procedure in such cases. The treatment of the young athletic patient with secondary impingement from instability focuses on restoring normal kinematics; however, it has been suggested that acromioplasty may have a role in some carefully selected younger patients with Type III acromions if primary impingement is clinically demonstrated. ${ }^{3}$

Codman ${ }^{7}$ referred to the region of the supraspinatus tendon where most tears initiate as the "critical portion." If impingement is an important early factor, subacromial contact would be expected to center on this area. Our finding that contact is indeed greatest on the distal supraspinatus tendon with the arm in elevation is consistent with a role for subacromial impingement in the pathogenesis of rotator cuff degeneration. Nasca et al. ${ }^{21}$ found a correlation between subacromial contact and areas of tendon damage. While this correlation does not establish impingement per se as the initiating etiology, it does suggest that a mechanical disturbance in subacromial gliding, such as increased soft tissue volume in the subacromial space, ${ }^{40}$ superior humeral subluxation because of rotator cuff dysfunction, ${ }^{15,16,34}$ superior humeral translation due to a tight posterior capsule,${ }^{18}$ or superior humeral subluxation due to glenohumeral instability, ${ }^{17}$ might be expected to selectively damage the supraspinatus insertion by focusing contact by the overlying anteroinferior acromion on this region.

The overhead, externally rotated arm position is a common starting point for many athletic activities. Rather than pick an arbitrary rotation, we chose the unique degree of external rotation (starting rotation) that allowed maximal elevation. This position minimized acromial proximity to the rotator cuff tendons. Therefore, factors that might keep the arm in a lesser degree of elevation, such as capsular tightness or apprehension from instability, may increase compression of the rotator cuff. Furthermore, actual overlap of the acromial and rotator cuff surfaces, implying deformation, occurred more frequently in specimens el- evated in $20^{\circ}$ less external rotation than the starting rotation (Table 1). If stiffness or apprehension from instability prevents full external rotation, it is possible that the rotator cuff tendon may be subjected to increased compressive loads. This lends support to treatment regimens emphasizing range of motion and elimination of stiffness.

\section{ACKNOWLEDGMENTS}

This work was supported in part by an Orthopaedic Research and Education Foundation Grant (CDA for ELF). The authors acknowledge the assistance of Craig Boulris, MD.

\section{REFERENCES}

1. Armstrong JR: Excision of the acromion in the treatment of the supraspinatus syndrome: Report of ninety-five excisions. I Bone Joint Surg 31B: 436-442, 1949

2. Ateshian GA, Soslowsky LJ, Mow VC: Quantitation of articular surface topography and cartilage thickness in knee joints using stereophotogrammetry. J Biomech 24: 761-776, 1991

3. Bigliani LU, D'Allesandro DF, Duralde XA, et al: Anterior acromioplasty for subacromial impingement in patients younger than 40 years of age. Clin Orthop 246: 111-116, 1989

4. Bigliani LU, Morrison DS, April EW: The morphology of the acromion and its relationship to rotator cuff tears. Orthop Trans 10:228, 1986

5. Bigliani LU, Ticker JB, Flatow EL, et al: The relationship of acromial architecture to rotator cuff disease. Clin Sports Med 10: 823-838, 1991

6. Burns WC II, Whipple TL: Anatomic relationships in the shoulder impingement syndrome. Clin Orthop 294: 96-102, 1993

7. Codman EA: The shoulder: Rupture of the supraspinatus tendon and other lesions in or about the subacromial bursa. Boston, Thomas Todd, 1934

8. Cone RO III, Resnick D, Danzig L: Shoulder impingement syndrome: Radiographic evaluation. Radiology 150: 29-33, 1984

9. Cotton RE, Rideout DR: Tears of the humeral rotator cuff: A radiological and pathological necropsy survey. J Bone Joint Surg 46B: 314-328, 1964

10. Doody SG, Freedman L, Waterland JC: Shoulder movements during abduction in the scapular plane. Arch Phys Med Rehabil 51: 595-604, 1970

11. Flatow EL, Fischer RA, Bigliani LU: Results of rotator cuff surgery, in lannotti J (ed): The Rotator Cuff. Chicago, AAOS, 1991

12. Freedman L, Munro RR: Abduction of the arm in the scapular plane: Scapular glenohumeral movements. J Bone Joint Surg 48A: 1503-1510, 1966

13. Golding FC: The shoulder: The forgotten joint. Br J Radiol 35: 149-158, 1962

14. Hawkins RJ, Brock RM, Abrams JS, et al: Acromioplasty for impingement with an intact rotator cuff. J Bone Joint Surg 70B: 795-797, 1988

15. Howard NJ, Eloesser L: Treatment of fracture of the upper end of the humerus. An experimental and clinical study. J Bone Joint Surg 32: 1-29, 1934

16. Jerosch J, Castro WHM, Sons HU, et al: Zur Atiologie des subacromialen Impingement-Syndromes: Eine biomechanische Untersuchung. Beitr Orthop Traumatol 36: 411-418, 1989

17. Jobe FW, Glousman RE: Rotator cuff dysfunction and associated glenohumeral instability in the throwing athlete, in Paulos LE, Tibone JE (eds): Operative Techniques in Shoulder Surgery. Gaithersburg, MD, Aspen, 1991, pp 85-91

18. Matsen FA III, Lippitt SB, Sidles JA, et al: In Practical Evaluation and Management of the Shoulder. Philadelphia, WB Saunders Co, 1994, pp 37-40

19. Meyer AW: Chronic functional lesions of the shoulder. Arch Surg 35: $646-674,1937$

20. Morrison DS, Bigliani LU: The clinical significance of variations in acromial morphology. Orthop Trans 11: 234, 1987

21. Nasca RJ, Salter EG, Weil CE: Contact areas of the subacromial joint, in Bateman JE, Welsh RP (eds); Surgery of the Shoulder. New York, Decker, 1984, p 134-139

22. Neer CS II: Impingement lesions. Clin Orthop 173: 70-77, 1983

23. Neer CS II: Anterior acromioplasty for the chronic impingement syndrome in the shoulder. J Bone Joint Surg 54A: 41-50, 1972

24. Neer CS II, Poppen NK: Supraspinatus outlet. Orthop Trans 11:234, 1987

25. Nevaiser TJ, Nevaiser RJ, Nevaiser JS: The four-in-one arthroplasty for the painful arc syndrome. Clin Orthop 163: 107-112, 1982

26. Nirschl RP: Rotator cuff tendinitis: Basic concept of pathoetiology. Instr Course Lect 38: 447-462, 1989

27. Perry J: Anatomy and biomechanics of the shoulder in throwing, swimming, gymnastics, and tennis. Clin Sports Med 2: 247-270, 1983

28. Petersson CJ, Redlund-Johnell I: The subacromial space in normal shoulder radiographs. Acta Orthop Scand 55: 52-58, 1984 
29. Poppen NK, Walker PS: Normal and abnormal motion of the shoulder. $J$ Bone Joint Surg 58A: 195-201, 1976

30. Post M, Cohen J: Impingement syndrome: A review of late stage II and early stage III lesions. Clin Orthop 207: 126-132, 1986

31. Regan WD, Richards RR: Subacromial pressure pre- and postacromioplasty: A cadaveric study. Orthop Trans 13: 671, 1989

32. Renoux $S$, Monet $J$, Pupin $P$, et al: Preliminary note on biometric data relating to the human coracoacromial arch. Surg Radiol Anat 8: 189-195, 1986

33. Scherrer PK, Hillberry BM, Van Sickle DC: Determining in vivo access of contact in the canine shoulder. J Biomech Eng 101: 271-278, 1979

34. Schwartz E, Warren RF, Otis JC, et al: Superior migration of the humeral head in rotator cuff dysfunction. Trans Orthop Res Soc 13: 444, 1988

35. Smith-Peterson MN, Aufranc OE, Larson CB: Useful surgical procedures for rheumatoid arthritis involving joints of the upper extremity. Arch Surg 46 . $764-770,1943$

36. Soslowsky LJ: Studies on diarthrodial joint biomechanics with special reference to the shoulder. PhD Thesis, Columbia University, New York, 1991

37. Soslowsky LJ, Ateshian GA, Mow VC: Stereophotogrammetric determination of joint anatomy and contact areas, in Mow SC, Ratcliffe A, Woo SL-Y (eds): Biomechanics of Diarthrodial Joints. Vol 2. New York, SpringerVerlag, 1990

38. Soslowsky LJ, Flatow EL, Bigliani LU, et al: Quantitation of in situ contact areas in the glenohumeral joint: A biomechanical study. $J$ Orthop Res 10: $524-534,1992$

39. Soslowsky LJ, Flatow EL, Pawluk RJ, et al: Subacromial contact (impingement) on the rotator cuff in the shoulder. Trans Orthop Res Soc 17: 424, 1992

40. Uhthoff HK, Hammond DI, Sarkar K, et al: The role of the coracoacromial ligament in the impingement syndrome. A clinical, radiological and histological study. Int Orthop 12: 97-104, 1988

41. Walch $G$, Boileau $P$, Noel $E$, et al: Impingement of the deep surface of the supraspinatus tendon on the posterosuperior glenoid rim: An arthroscopic study. J Shoulder Elbow Surg 1: 238-245, 1992

42. Warren RF: Surgical considerations of rotator cuff tears in athletes, in Jackson DW (ed): Shoulder Surgery in the Athlete. Rockville, MD, Aspen, 1985, pp 73-82

43. Watson-Jones R: Fractures and Joint Injuries. Fifth edition. Vol 2. London, Churchill Livingstone, 1976, pp 524-527

44. Weiner DS, MacNab I: Superior migration of the humeral head: A radiological aid in the diagnosis of tears of the rotator cuff. J Bone Joint Surg 52B: 524-527, 1970

45. Zuckerman JD, Kummer FJ, Cuomo F, et al: The influence of coracoacromial arch anatomy on rotator cuff tears. J Shoulder Elbow Surg 1: 4-14, 1992 